Comparative Corporate Governance 



\title{
Comparative Corporate Governance
}

- Essays and Materials -

\author{
Edited by \\ Klaus J. Hopt \\ Director of the Max-Planck-Institute \\ for Foreign Private and Private International Law, \\ Hamburg, Germany \\ Eddy Wymeersch \\ Professor at the University of Ghent, Belgium \\ Director of the Seminar of Commercial and Enterprise Law
}

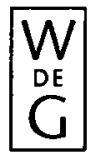

1997

Walter de Gruyter - Berlin · New York 
Comparative corporate governance : essays and materials / edited by Klaus J. Hopt and Eddy Wymeersch.

XIV, 356 p. $15,5 \times 23,0 \mathrm{~cm}$.

"This book has grown out of a conference entitled "Comparative Corporate Governance, an International Conference, United States - Japan - Western Europe' which was held in Brussels on 14 June 1995" - Pref.

English with some contributions in French and German.

ISBN 3-11-015765-9

1. Corporate governance - Congresses. 2. Comparative management - Congresses. 3. Corporate governance - Law and legislation - Congresses. 4. Comparative law - Congresses. I. Hopt, Klaus J., 1940 - . II. Wymeersch, E. HD2741.C6247 1997 $658.4-\mathrm{dc} 21$

Die Deutsche Bibliotbek - CIP-Einheitsaufrabme

Comparative corporate governance : essays and materials / ed. by Klaus J. Hopt and Eddy Wymeersch. - Berlin ; New York : de Gruyter, 1997

ISBN 3-11-015765-9

(C) Copyright 1997 by Walter de Gruyter \& Co., D-10785 Berlin

All rights reserved, including those of translation into foreign languages. No part of this book may be reproduced or transmitted in any form or by any means, electronic or mechanical, including photocopy, recording, or any information storage and retrieval system, without permission in writing from the publisher.

Printing: WB-Druck, D-87669 Rieden am Forggensee

Binding: Lüderitz \& Bauer GmbH, D-10963 Berlin Printed in Germany. 